\title{
Temperature-dependent Raman scattering spectra of $\varepsilon$-GaSe layered crystal
}

\author{
N.M. Gasanly ${ }^{\mathrm{a}, *, 1}$, A. Aydınlı ${ }^{\mathrm{b}}$, H. Özkan ${ }^{\mathrm{a}}$, C. Kocabaş ${ }^{\mathrm{b}}$ \\ ${ }^{a}$ Physics Department, Middle East Technical University, 06531 Ankara, Turkey \\ ${ }^{\mathrm{b}}$ Physics Department, Bilkent University, 06533 Ankara, Turkey
}

(Refereed)

Received 2 May 2001; received in revised form 22 October 2001; accepted 9 November 2001

\begin{abstract}
The temperature dependencies $(15-300 \mathrm{~K})$ of seven Raman-active mode frequencies and linewidths in layered gallium selenide have been measured in the frequency range from 10 to $320 \mathrm{~cm}^{-1}$. We observed softening and broadening of the optical phonon lines with increasing temperature. Comparison between the experimental data and theories of the shift and broadening of the intralayer phonon lines during heating of the crystal showed that the experimental dependencies can be explained by the contributions from thermal expansion, lattice anharmonicity and crystal disorder. The pure-temperature contribution (phononphonon coupling) is due to three-phonon processes. Moreover, it was established that the effect of crystal disorder on the linewidth broadening of TO mode is stronger than that of LO mode. (C) 2002 Elsevier Science Ltd. All rights reserved.
\end{abstract}

PACS: 78.20. -e; 78.30. -j; 78.30. Hv

Keywords: A. Chalcogenides; B. Semiconductors; C. Raman spectroscopy; D. Optical properties

\section{Introduction}

$\mathrm{A}^{\mathrm{III}} \mathrm{B}^{\mathrm{VI}}$-type semiconducting compounds, GaSe, GaS, and InSe, crystallize in a layered structure. These layered compounds are characterized by a highly anisotropic bonding forces. The high anisotropy arises from the fact that the bondings within the

\footnotetext{
${ }^{*}$ Corresponding author. Fax: +90-312-210-1281.

E-mail address: nizami@metu.edu.tr (N.M. Gasanly).

${ }^{1}$ On leave from Physics Department, Baku State University, Baku, Azerbaijan.
} 
layers are considerably stronger than those perpendicular to them. In GaSe, van der Waals forces contribute predominantly to the interlayer interaction, while the bonding forces within the layers are primarily covalent. Because of the extremely weak interlayer interaction, a GaSe crystal can be easily cleaved along the layer.

The lattice vibrations of GaSe crystal have been studied using Raman scattering [16], infrared reflectivity and absorption [3,7], inelastic neutron scattering [8], and Brillouin scattering measurements [9]. Room temperature Raman scattering spectra of GaSe crystal are measured under pressure up to $8 \mathrm{GPa}$, and the pressure coefficients for all the Raman-active modes are obtained [10].

The influence of anharmonic interactions on the lattice vibrations can be experimentally studied by measuring changes of phonon frequency and linewidth with temperature and pressure. A large number of articles devoted to the study of the temperature dependence of the frequency shift and the linewidth of the first-order Raman scattering in the semiconductors may be found in the literature [11-16]. They show that the Raman shift can be successfully modeled by including the effect of thermal expansion and the phonon-phonon coupling.

The aim of the present study was to measure the frequency and linewidth, fullwidth at half-maximum (FWHM), of optical phonons in $\varepsilon$-GaSe layered crystals from the Raman spectra in the temperature range from 15 to $300 \mathrm{~K}$. We report softening and broadening of the optical phonon lines at the Brillouin zone center with increase of temperature as observed in some other semiconductors. Our analysis and results indicate that the purely anharmonic contribution to the phonon frequency shift and linewidth broadening are due to interaction with phonons of other branches. Moreover, we studied the effect of crystal disorder on the linewidth broadening of transverse optical (TO) and longitudinal optical (LO) phonons.

\section{Experimental}

Gallium selenide polycrystals were synthesized from high-purity elements (at least 99.999\%) taken in stoichiometric proportions. GaSe single crystals were grown by the modified Bridgman method. The analysis of X-ray diffraction data showed that they crystallize in hexagonal unit cell with parameters: $a=0.3745 \mathrm{~nm}$ and $c=$ $1.5921 \mathrm{~nm}$. Crystals suitable for measurements were obtained by easy cleavage perpendicular to optical $c$-axis. As grown GaSe is an p-type semiconductor having an indirect band gap with energies of 2.065 and $2.075 \mathrm{eV}$ at 77 and $4.2 \mathrm{~K}$, respectively [17].

Unpolarized Raman scattering measurements in $\varepsilon$-GaSe layered crystal were performed in the back-scattering geometry in the frequency range $10-320 \mathrm{~cm}^{-1}$. Thirty milliwatt He-Ne laser $(632.8 \mathrm{~nm})$ was used as the exciting light source. The scattered light was analyzed using a U-1000 "Jobin Yvon" double grating spectrometer and a cooled GaAs photomultiplier supplied with necessary photon counting electronics. The Raman line positions were determined within an accuracy of $\pm 0.1 \mathrm{~cm}^{-1}$. A "CTI-Cryogenics M-22" closed-cycle helium cryostat was used to 
cool the crystals from room temperature down to $15 \mathrm{~K}$. The temperature was controlled within an accuracy of $\pm 0.5 \mathrm{~K}$.

To achieve high signal-to-noise ratio $(>100)$, we should keep the slit widths in the spectrometer equal to at least $200 \mu \mathrm{m}$. Below $200 \mu \mathrm{m}$, the signal-to-noise ratio was so small that we could not measure the linewidth of the phonon lines with high accuracy. Since the measured phonon lines of GaSe crystal were very narrow, one had to correct them for the finite instrumental resolution. The width of the response function of the spectrometer was determined by measuring the width of the laser line with the same slit apertures as in the Raman experiment. With the slit widths of $200 \mu \mathrm{m}$, we could obtain an instrumental resolution of $1.16 \mathrm{~cm}^{-1}$. The observed peak is the convolution of the Lorentzian shape of the actual phonons with the response function of the spectrometer, which is considered to be a Gaussian. The convolution product of a Gaussian times Lorentzian curve is the so-called Voigt profile. To make the deconvolution, at first we fit a Voigt profile to our experimental peaks, and then calculate the Lorentzian linewidth using the fitted width of the Voigt profile and the experimentally determined width of the spectrometer response function.

\section{Results and discussion}

$\varepsilon$-Polytype of GaSe has two layers in the unit cell. Each layer consists of four sheets of atoms stacked along the $c$-axis in the sequence $\mathrm{Se}-\mathrm{Ga}-\mathrm{Ga}-\mathrm{Se}$. For $\varepsilon-\mathrm{GaSe}, 24$ normal modes at the Brillouin zone center are given by the irreducible representations of the $D_{3 \mathrm{~h}}$ point group as

$$
\Gamma \equiv 4 A_{1}^{\prime}+4 A_{2}^{\prime \prime}+4 E^{\prime}+4 E^{\prime \prime}
$$

In this case, there are 11 non-degenerate Raman-active modes $\left(4 A_{1}^{\prime}+3 E^{\prime}+4 E^{\prime \prime}\right)$ and six IR-active modes $\left(3 A_{2}^{\prime \prime}+3 E^{\prime}\right)$. Ten Raman-active modes form five Davydov pairs $\left(2 A_{1}^{\prime}, 2 A_{1}^{\prime}, 2 E^{\prime}, 2 E^{\prime \prime}, 2 E^{\prime \prime}\right)$. One of the Raman-active $3 E^{\prime}$ modes $\left(E^{\prime(2)}\right)$ and acoustic mode $E^{\prime(1)}$ are counterparts of the sixth Davydov doublet [2]. The $\varepsilon$-GaSe lacks an inversion center. Therefore, both Raman- and IR-active modes (both active modes) are allowed which are split into TO and LO modes by long-range Coulomb interaction. Thus, in addition to the TO mode, the LO mode can be observed in the Raman spectrum of $\varepsilon-\mathrm{GaSe}[1-3,6]$.

We used Melvin projection operator method [18] to obtain the displacement vectors of atoms in all phonon modes. Fig. 1 shows the atomic displacement vectors for interlayer and intralayer optical modes of $\varepsilon$-GaSe crystal. As seen from Fig. 1, in these modes, all the gallium and selenium atoms move either perpendicular or parallel to the layers.

Fig. 2 represents the comparison of the Raman spectra of $\varepsilon-\mathrm{GaSe}$ at 15 and $300 \mathrm{~K}$. The shift and broadening of seven phonon lines with increasing temperature are clearly seen. The present assignment of the observed modes is in excellent agreement with that of [1]. In addition, doublet has been observed for $E^{\prime(4)}$ (TO) mode. We have measured and analyzed the interlayer and intralayer optical modes with room 


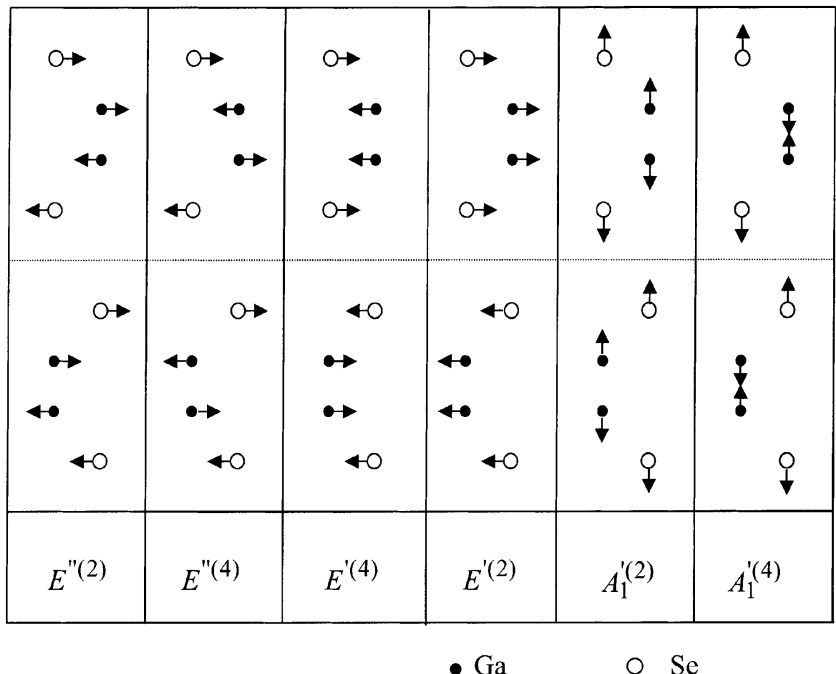

Fig. 1. Atomic displacement vectors for interlayer and intralayer Raman-active optical modes of $\varepsilon$-GaSe crystal.

temperature frequency values $19.7\left(E^{\prime(2)}\right), 59.4\left(E^{\prime \prime(2)}\right), 209.7\left(E^{\prime \prime(4)}\right), 212.8$ and 214.2 $\left(E^{\prime(4)}(\mathrm{TO})\right), 253.2\left(E^{\prime(4)}(\mathrm{LO})\right), 134.1\left(A_{1}^{\prime(2)}\right)$, and $307.8\left(A_{1}^{\prime(4)}\right) \mathrm{cm}^{-1}$. As seen from Fig. 1 , in the $E^{\prime(2)}, E^{\prime \prime(4)}, E^{\prime(4)}, E^{\prime \prime(2)}$ shear modes the atoms vibrate in the direction of bending bonds, whereas the $A_{1}^{\prime(2)}$ and $A_{1}^{\prime(4)}$ compressional modes correspond to the

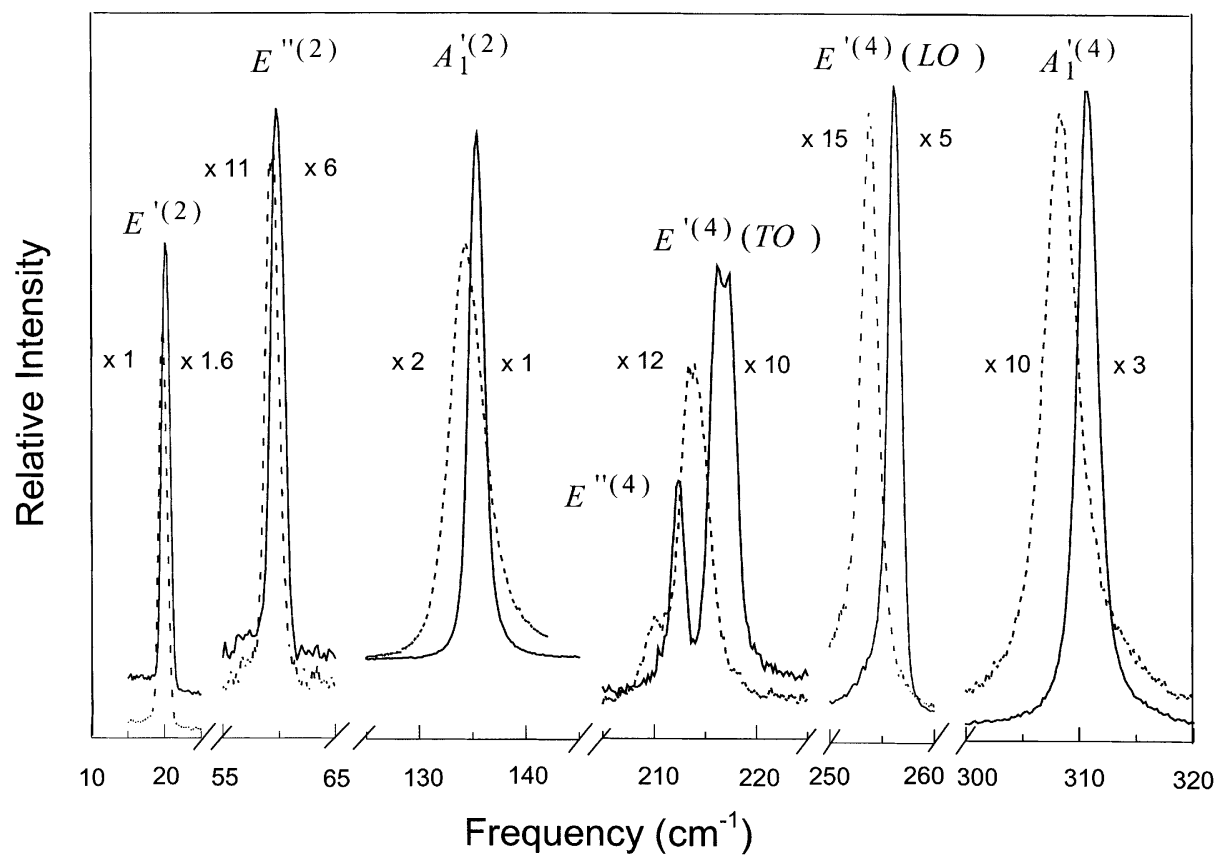

Fig. 2. Comparison of the extended individual parts of Raman spectra of GaSe crystal at $T=15 \mathrm{~K}(-)$ and $300 \mathrm{~K}(---)$. 
stretching vibrations of the atoms. The interlayer shear mode $E^{\prime(2)}$ in which entire layers vibrate rigidly out of phase with their neighbors relates only to the weak layerlayer interaction. The low value of this mode frequency $\left(v=19.7 \mathrm{~cm}^{-1}\right)$ gives information about the strength of the layer-layer interaction in GaSe. Gauthier et al. [10] previously observed that all mode frequencies of GaSe crystal increase with pressure. It was reported that there is a large difference between the mode Grüneisen parameter $(\gamma)$ of the interlayer shear mode $E^{\prime(2)}(22.1)$ and the $\gamma$ values of the intralayer shear $\left(E^{\prime \prime(2)}(1.3), E^{\prime \prime(4)}(1.9), E^{\prime(4)}\right.$ (TO) (1.8), $E^{\prime(4)}$ (LO) (1.1)) and compressional $\left(A_{1}^{\prime(2)}(3.9)\right.$ and $\left.A_{1}^{\prime(4)}(1.4)\right)$ modes which represents the difference in the interlayer and intralayer restoring forces. The total frequency shifts of GaSe Raman modes in the temperature range $15-300 \mathrm{~K}$ were found to be $0.6\left(E^{\prime(2)}\right), 0.4$ $\left(E^{\prime \prime(2)}\right), 2.5\left(E^{\prime \prime(4)}\right), 3.3$ and $3.2\left(E^{\prime(4)}(\mathrm{TO})\right), 3.0\left(E^{\prime(4)}(\mathrm{LO})\right), 1.4\left(A_{1}^{\prime(2)}\right)$ and 2.9 $\left(A_{1}^{\prime(4)}\right) \mathrm{cm}^{-1}$. Analysis of the temperature dependence of the frequency shift for the low-frequency interlayer mode $E^{\prime(2)}$ does not yield a physically meaningful decay channel. This is consistent with the narrow linewidth of this mode which indicates a long lifetime. Fig. 3 shows the experimental results (open circles) for the line positions $v(T)$ of one of the intralayer modes, $\left(A_{1}^{\prime(4)}\right)$. The phonon frequency shift with temperature can be described by the expression $[11,13,19]$

$$
v(T)=v_{0}+\Delta_{1}(T)+\Delta_{2}(T),
$$

where $v_{0}+\Delta_{2}(0)$ is the Raman shift as $T$ approaches $0 \mathrm{~K}, \Delta_{1}(T)$ represents the volume dependence of the frequency due to the thermal expansion of the crystals

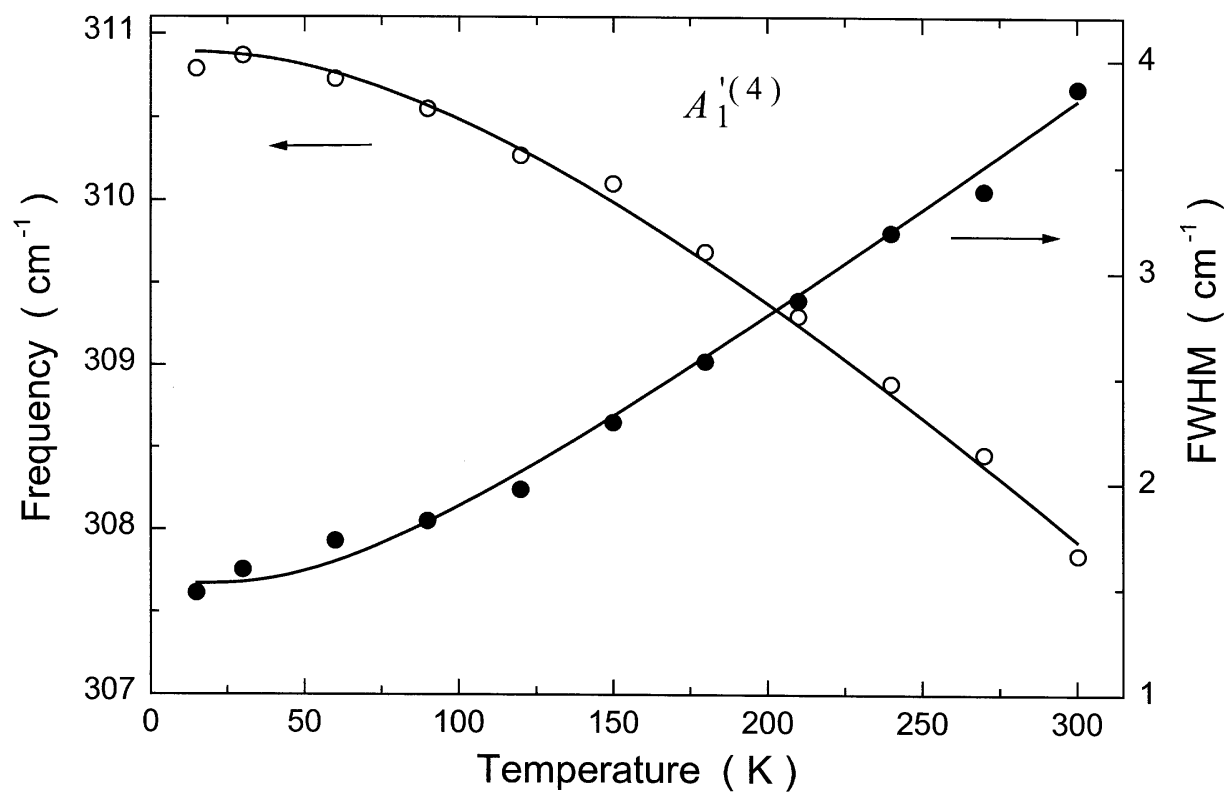

Fig. 3. Temperature dependencies of the $A_{1}^{\prime(4)}$ intralayer mode frequency $(\bigcirc)$ and linewidth $(\bigcirc)$ in GaSe crystal. The solid curves, frequency and FWHM, give the theoretical fits using Eqs. (1)-(3) for frequency and Eq. (4) for FWHM. 
and $\Delta_{2}(T)$ specifies the contribution of anharmonic coupling to phonons of other branches.

$\Delta_{1}(T)$ can be written as

$$
\Delta_{1}(T)=v_{0}\left[\exp \left(-3 \gamma \int_{0}^{T} \alpha\left(T^{\prime}\right) \mathrm{d} T^{\prime}\right)-1\right],
$$

where $\alpha(T)$ is the coefficient of linear thermal expansion.

The purely anharmonic contribution to the frequency shift can be modeled as

$$
\Delta_{2}(T)=A\left[1+\frac{1}{\mathrm{e}^{x_{1}}-1}+\frac{1}{\mathrm{e}^{x_{2}}-1}\right],
$$

which represents the coupling of the optical phonon to two different phonons (threephonon processes). Here $x_{1}=h c v_{1} / k_{\mathrm{B}} T$ and $x_{2}=h c v_{2} / k_{\mathrm{B}} T$. The experiments in the present study were carried out at temperatures below the Debye temperature of GaSe crystal $\left(\theta_{\mathrm{D}}=342 \mathrm{~K}[8]\right)$, thus the three-phonon process is dominant and the higher order processes can be neglected.

Using the experimental values of $\gamma$ [10] and $\alpha(T)$ [20], the frequency shifts for intralayer modes were fitted by means of Eqs. (1)-(3) with $A, v_{0}, v_{1}$, and $v_{2}$ as adjustable parameters, with keeping the sum of $v_{1}+v_{2}=v_{0}$ constant (energy conservation). For all intralayer modes, the agreement between the theoretical and experimental dependencies was found to be good. Fig. 3 shows this agreement for one representative $\left(A_{1}^{\prime(4)}\right)$ of the intralayer modes. The resulting parameters for all the intralayer modes are shown in Table 1 . The frequencies $v_{1}$ and $v_{2}$ allowed by energy conservation $\left(v_{1}+v_{2}=v_{0}\right)$ for Raman-active modes are present on phonon dispersion curves of $\varepsilon$-GaSe in $\left\langle\begin{array}{lll}1 & 0 & 0\end{array}\right\rangle$ direction [8]. Thus, we have determined the following decay channels which satisfy conservation laws: $E^{\prime \prime(2)} \rightarrow \mathrm{TA}+A_{2}^{\prime \prime} ; A_{1}^{\prime(2)} \rightarrow \mathrm{LA}+$ $E^{\prime \prime} ; \quad E^{\prime \prime(4)} \rightarrow \mathrm{TA}+E^{\prime \prime} ; \quad E^{\prime(4)}(\mathrm{TO}) \rightarrow \mathrm{LA}+A_{1}^{\prime} ; \quad E^{\prime(4)}(\mathrm{LO}) \rightarrow \mathrm{TA}+A_{2}^{\prime \prime} ; \quad A_{1}^{\prime(4)} \rightarrow$ $E^{\prime \prime}+A_{2}^{\prime \prime}$. These results indicate that the pure-temperature dependence of the frequencies $\Delta_{2}(T)$ is dominated by the three-phonon processes.

The linewidth of the GaSe phonons was also studied systematically as a function of temperature in the range of $15-300 \mathrm{~K}$. The measured linewidths of interlayer mode

Table 1

Parameters for fitting the temperature dependencies of Raman frequency and linewidth of the GaSe crystal

\begin{tabular}{lccclll}
\hline Modes & $v_{0}\left(\mathrm{~cm}^{-1}\right)$ & $v_{1}\left(\mathrm{~cm}^{-1}\right)$ & $v_{2}\left(\mathrm{~cm}^{-1}\right)$ & $A\left(\mathrm{~cm}^{-1}\right)$ & $\Gamma_{0}\left(\mathrm{~cm}^{-1}\right)$ & $C\left(\mathrm{~cm}^{-1}\right)$ \\
\hline$E^{\prime \prime(2)}$ & 59.9 & 22.0 & 37.9 & -0.01 & - & - \\
$A_{1}^{\prime(2)}$ & 135.2 & 55.2 & 80.0 & -0.19 & 0.33 & 0.49 \\
$E^{\prime \prime(4)}$ & 212.5 & 70.9 & 141.6 & -0.28 & 0.11 & 0.26 \\
$E^{\prime \prime(2)}(\mathrm{TO})$ & 216.5 & 82.3 & 134.2 & -0.46 & 0.68 & 0.28 \\
& 217.8 & 82.7 & 135.1 & -0.41 & 0.68 & 0.28 \\
$E^{\prime(4)}(\mathrm{LO})$ & 256.7 & 75.3 & 181.4 & -0.52 & 0.44 & 0.31 \\
$A_{1}^{\prime(4)}$ & 311.4 & 109.4 & 202.0 & -0.46 & 0.46 & 1.07 \\
\hline
\end{tabular}


$\left.E^{\prime 2}\right)\left(1.29 \mathrm{~cm}^{-1}\right)$ and low-frequency intralayer mode $E^{\prime \prime(2)}\left(1.32 \mathrm{~cm}^{-1}\right)$ at low temperatures became comparable to that of the instrument $\left(1.16 \mathrm{~cm}^{-1}\right)$. Therefore, we have not analyzed the temperature dependence of the linewidth of these two modes. The corrected linewidths of intralayer Raman modes at room temperature were found to be $1.1\left(E^{\prime \prime(4)}\right), 1.9$ and $1.9\left(E^{\prime(4)}(\mathrm{TO})\right), 1.7\left(E^{\prime(4)}(\mathrm{LO})\right), 3.8\left(A_{1}^{\prime(2)}\right)$, and $3.9\left(A_{1}^{\prime}{ }^{(4)}\right) \mathrm{cm}^{-1}$. The linewidth of all optical modes are found to increase with temperature. The temperature dependence of $A_{1}^{\prime(4)}$ mode linewidth (solid circles) is shown in Fig. 3. The broadening of the phonon lines is due to anharmonicity of the lattice vibrations. The presence of anharmonic forces in a crystal lead to interactions between the harmonic normal modes that produce a temperature-dependent lifetime of the normal modes.

The temperature dependence of the phonon linewidth can be described as follows $[11,13,19,21]$ :

$$
\Gamma=\Gamma_{0}+C\left[1+\frac{1}{\mathrm{e}^{x_{1}}-1}+\frac{1}{\mathrm{e}^{x_{2}}-1}\right],
$$

where $\Gamma_{0}$ is the temperature-independent broadening due to the disorder of crystal and $C$ is the broadening of the phonon line due to the cubic anharmonicity at absolute zero (the decrease in phonon lifetime $\tau$ due to the decay of the optical phonon into two different phonons).

The experimental data of phonon linewidth for intralayer modes of GaSe were fitted by means of Eq. (4) with $\Gamma_{0}, C, v_{1}$, and $v_{2}$ as fitting parameters, while keeping the sum of $v_{1}+v_{2}=v_{0}$ constant (solid curve in Fig. 3). As seen from this figure, we obtain quantitative agreement between calculated curve and experimental points for intralayer $A_{1}^{\prime(4)}$ mode. The fitting parameters for all the intralayer modes are presented in Table 1. The linewidth broadenings due to the crystal disorder were found to be 0.68 and $0.44 \mathrm{~cm}^{-1}$ for TO and LO modes, respectively. The parameter $\Gamma_{0}$ for LO mode is small compared to that for TO mode. Similar results were previously obtained by Anand et al. [13] and Bairamov et al. [22] for ZnSe and GaP crystals, respectively. The simple model presented here shows that the cubic anharmonicity accounts well for the temperature dependence of the linewidth of the optical phonons studied.

\section{Conclusions}

The analysis of the temperature dependence of the optical modes in GaSe crystal shows that the Raman frequency shift and broadening of linewidths are well described by considering the thermal-expansion, pure-temperature (phonon-phonon coupling) and crystal disorder contributions. The cubic (three-phonon) anharmonicity with energy conservation, responsible for the pure-temperature contributions to the softening and broadening of the phonon lines with increasing temperature, was determined. It was established that the effect of crystal disorder on the linewidth broadening of TO mode is stronger than that of LO mode $\left(\Gamma_{0}=0.68\right.$ and $0.44 \mathrm{~cm}^{-1}$, respectively). 


\section{References}

[1] J.C. Irwin, R.M. Hoff, B.P. Clayman, R.A. Bromley, Solid State Commun. 13 (1973) 1531.

[2] H. Yoshida, S. Nakashima, A. Mithsuitshi, Phys. Stat. Sol. (b) 59 (1983) 655.

[3] N.M. Gasanly, A.F. Goncharov, N.N. Melnik, A.S. Ragimov, Phys. Stat. Sol. (b) 120 (1983) 137.

[4] A. Polian, K. Kunc, A. Kuhn, Solid State Commun. 19 (1976) 1079.

[5] V.V. Artamonov, M.Y. Valakh, M.P. Lisitsa, Phys. Stat. Sol. (b) 105 (1981) K103.

[6] K.R. Allakhverdiev, E.Y. Salaev, M.M. Tagyev, S.S. Babaev, L. Genzel, Solid State Commun. 59 (1986) 133.

[7] V. Riede, H. Neumann, H. Sobotta, F. Levy, Solid State Commun. 34 (1980) 229.

[8] S. Jandl, J.L. Brebner, B.M. Powell, Phys. Rev. B 13 (1976) 686.

[9] Y. Honma, M. Yamada, K. Yamamoto, K. Abe, J. Phys. Soc. Jpn. 52 (1983) 2777.

[10] M. Gauthier, A. Polian, J.M. Besson, A. Chevy, Phys. Rev. B 40 (1989) 3837.

[11] J. Menendez, M. Cardona, Phys. Rev. B 29 (1984) 2051.

[12] P. Verma, S.C. Abbi, K.P. Jain, Phys. Rev. 51 (1995) 16660.

[13] S. Anand, P. Verma, K.P. Jain, S.C. Abbi, Physica B 226 (1996) 331.

[14] J. Gonzalez, E. Moya, J.C. Chervin, Phys. Rev. B 54 (1996) 4707.

[15] J. Gonzalez, Y. Guinet, J. Lefebvre, Cryst. Res. Technol. 31 (1996) 453.

[16] G. Irmer, M. Wenzel, J. Monecke, Phys. Stat. Sol. (b) 195 (1996) 85.

[17] E. Aulich, J.L. Brebner, E. Mooser, Phys. Stat. Sol. (b) 31 (1969) 129.

[18] M.A. Melvin, Rev. Mod. Phys. 28 (1956) 18.

[19] A. Debernardi, Solid State Commun. 113 (2000) 1.

[20] G.L. Belenkii, R.A. Suleymanov, N.A. Abdullaev, V.Y. Shteinschraiber, Sov. Phys. Solid State 26 (1984) 2142.

[21] A. Debernardi, M. Cardona, Physica B 263/264 (1999) 687.

[22] B.K. Bairamov, Y.E. Kitaev, V.K. Negoduiko, Z.M. Khashkhozhev, Sov. Phys. Solid State 16 (1975) 1323. 